\title{
Transient Spin Dynamics in Semiconductors
}

\author{
L. Villegas-Lelovsky \\ Departamento de Física e Informática, Instituto de Física de São Carlos, \\ Universidade de São Paulo, 13560-970 São Carlos, São Paulo, Brazil
}

Received on 8 December, 2005

\begin{abstract}
We investigate the spin-resolved dynamics of spin-polarized carriers injected via a ferromagnetic scanningtunnelling-microscope tip (STM tip) into uniformly and non-uniformly n-doped bulk semiconductor - externally driven by a current source. We propagate the injected spin packets (assumed gaussian in space at $t=0$ ) by considering a spin hydrodynamic approach based on balance equations directly derived from a spin-dependent Boltzmann equation. We determine the spin-polarization landscapes (time and position) of the carrier population $\left(n_{\uparrow}-n_{\downarrow}\right) /\left(n_{\uparrow}+n_{\downarrow}\right)$ and the current density $\left(j_{\uparrow}-j_{\downarrow}\right) /\left(j_{\uparrow}+j_{\downarrow}\right)$. While in the uniformly-doped system the carrier spin-polarization has a slow decay, in the non-uniformly doped system it shows a drastic fall down in the interface. In contrast the current spin-polarization exhibits an enhancement for both of the systems particularly in the interface.
\end{abstract}

Keywords: Spin polarized transport; Semiconductors; Interfaces; Electrical injection

\section{INTRODUCTION}

The dynamics of photogenerated carriers in semiconductors and their heterostructures is a mature subject. A variety of interesting time-dependent phenomena have been investigated over the past decade or so: coupled plasmon-phonon dynamics, coherent phonon oscillations, terahertz emission from bulk systems pumped with ultrashort laser pulses, etc. Strictly speaking, the complex carrier dynamics of a semiconductor system following pump excitation should require a full-blow non-equilibrium calculation (e.g., via the Keldysh formalism) - particularly if one is interested in the quantumcoherent effects of the transient dynamics of electrons, holes, and phonons. Other approaches in terms of the semiclassical Boltzmann equation can provide a suitable description for diffusive transport dynamics. A hydrodynamic approach based on the moments of the Boltzmann equation - i.e., the continuity equations for the particle and the current densities - is yet another possibility. Recently, this simpler approach has been used to successfully describe the terahertz carrier-phonon dynamics and the corresponding electromagnetic emission from bulk semiconductors [1]

Motivated by the recent realization of ferromagnetic STM tip injection [2], we investigate in this work the transient dynamics of spin-polarized electron packets STM-tip injected into a $n$-doped GaAs semiconductor, Fig. 1. We solve the particle- and current-density continuity equations - equations involving the first and the second moments of the spinresolved Boltzmann equation - coupled to Poisson's equation describing the internal electric field profile. Spin-flip and momentum-relaxation processes are taken into account within the average relaxation-time approximation [3]. Our spinresolved hydrodynamic description goes beyond the usual drift-diffusion type approaches [4], [5], [6], [7] in that we fully account for the temporal evolution of the current densities. For STM tip injection into a uniformly doped $n$-GaAs system and into a $n / n+$ GaAs junction driven by a current source, we find the spin-polarization landscapes for carrier and current densities.

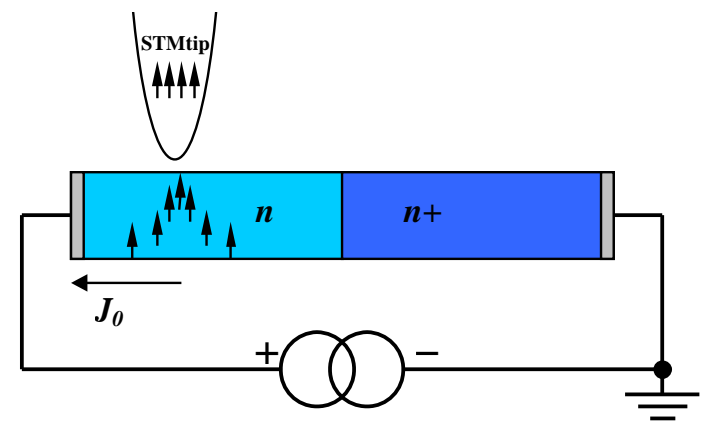

FIG. 1: Schematic of the ferromagnetic STM-tip spin injection into a inhomogeneous $n / n^{+}$GaAs, externally driven by a current source $J_{0}$. We also consider injection into uniformly $n$-doped bulk GaAs (not shown). The injection profile is assumed gaussian in space at $\delta t \leq 0.1 \mathrm{ps}$. By numerically propagating our continuity equations, we determine the spin-resolved carrier and current densities and the corresponding spin-polarization landscapes [Figs. 2(a)-(d)].

\section{SPIN HYDRODYNAMIC EQUATIONS}

The starting point of our approach is the spin-dependent Boltzmann equation governing the evolution of the semiclassical distribution function $f^{\sigma}(\mathbf{r}, \mathbf{k}, t)$ of the carriers with spin component $\sigma=\uparrow, \downarrow(\bar{\sigma}=\downarrow, \uparrow)$ in a bulk semiconductor. The number of the carriers with spin component $\sigma$ in a volume $d \mathbf{r} d \mathbf{k}$ about the phase-space point $(\mathbf{r}, \mathbf{k})$ is $f^{\sigma}(\mathbf{r}, \mathbf{k}, t) d^{3} r d^{3} k$. Following the usual hydrodynamic approach [8], we derive one-dimensional continuity equations for the spin-dependent carrier $n^{\sigma}(x, t)=\sum_{\mathbf{k}} f_{\mathbf{k}}^{\sigma}$ and the current densities $J^{\sigma}(x, t)=$ $\sum_{\mathbf{k}} f_{\mathbf{k}}^{\sigma} k_{x}$ from the corresponding moments of the spin Boltzmann equation [9]. Within the average relaxation time approximation and by assuming thermal equilibrium with a constant temperature $T$, we are able to close the hierarchy of the 
coupled moments thus obtaining the finite set of equations

$$
\begin{gathered}
\frac{\partial n^{\sigma}}{\partial t}+\frac{\partial J^{\sigma}}{\partial x}=G^{\sigma}-\frac{n^{\sigma}-n^{\bar{\sigma}}}{\tau_{s f}}, \\
\frac{\partial J^{\sigma}}{\partial t}+\frac{\partial}{\partial x}\left(\frac{J}{n} J^{\sigma}\right)= \\
\frac{q E}{m_{e}^{*}} n^{\sigma}-\frac{k_{B} T}{m_{e}^{*}} \frac{\partial}{\partial x} n^{\sigma}-\frac{J^{\sigma}}{\tau^{\sigma}}-\frac{J^{\sigma}-J^{\bar{\sigma}}}{\tau_{s f}^{*}} .
\end{gathered}
$$

where $q=-e$ is the electron charge and $m_{e}^{*}$ is the electron effective mass, which describes the evolution of our system. The three distinct average relaxation times in Eqs. (1) and (2) denote (i) the spin-dependent momentum relaxation time $\tau^{\sigma}$, (ii) the intrinsic carrier spin-flip time $\tau_{s f}$ describing the equilibration of the spin populations, and (iii) the current-density spin-flip time $\tau_{s f}^{*}$ representing the tendency of equalization of the two spin-channel current densities through momentum scattering with spin flip [10]. The total carrier and current densities are $n=n^{\sigma}+n^{\bar{\sigma}}$ and $J=J^{\sigma}+J^{\bar{\sigma}}$. We have added a generation term $G^{\sigma}(x, t)$ (gaussian in space and a delta function in time) to the continuity equation (1), representing the injection of spin-oriented carriers (e.g., via ferromagnetic STM tip or optical generation with circularly polarized light). In the case of non-magnetic semiconductors $\tau^{\sigma} \rightarrow \tau$ and we recover the spinless continuity equations of Ref.[1] by adding the two spin resolved components in Eq. (1).

\section{POISSON'S EQUATION}

The internal electric field $E(x, t)$ is calculated via,

$$
\frac{\partial E(x, t)}{\partial x}=\frac{q}{\varepsilon_{0} \varepsilon_{\infty}}\left[n^{\sigma}(x, t)+n^{\bar{\sigma}}(x, t)-n_{d}(x)\right]
$$

where $\varepsilon_{0}$ is the vacuum permeability constant, $\varepsilon_{\infty}$ is the dielectric constant, $n_{d}(x)$ is ionized donor concentration.

\section{NUMERICAL CALCULATION}

We develop numerical calculations via finite-differences discretization. By propagating the resulting discretized equations in time and space via explicit schemes (Lax method) [11], we calculate the carrier- and current-density profiles and from these we determine the corresponding spin-polarization landscapes, Figs. 2(a)-(d). In our simulations we use $n_{d}(x)=$ $10^{17} \mathrm{~cm}^{-3}(0 \leq x \leq 1 \mu \mathrm{m})$ ( $n$ GaAs bulk case) and $n_{d}(x)=$ $10^{17} \mathrm{~cm}^{-3}(0 \mu \mathrm{m} \leq x \leq 0.5 \mu \mathrm{m}), 5 \times 0{ }^{17} \mathrm{~cm}^{-3}(0.5 \mu \mathrm{m}<x \leq 1 \mu \mathrm{m})$ $\left(n / n+\right.$ GaAs junction case) $T_{e}=300 \mathrm{~K}$, an external electric field $E_{0}=-10 \mathrm{kV} / \mathrm{cm}, \tau=100 \mathrm{fs}[12], \tau_{s f}=4$ ps [13], $\left.\tau_{s f}^{*} \gg \tau_{s f}[14]\right)$. The 1D study is performed by assuming that the important features of the charge transport in the system are given in the electric field direction.
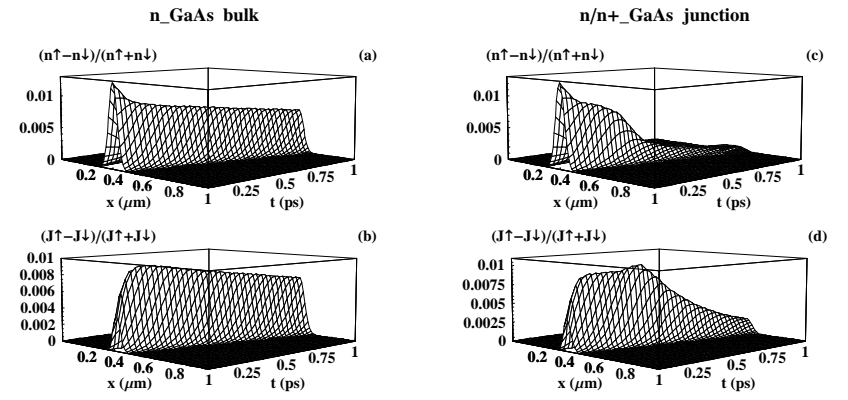

FIG. 2: Spin-polarization landscapes of the carrier and current densities for homogeneous (a) and inhomogenous (b) doped GaAs after propagating a Gaussian-like spin-up polarized pulse of electrons injected in $x_{0}=0.4 \mu \mathrm{m}$ by a fm-STM tip. While the carrier spin-polarization (Fig. 2(c)) is lost at interface the current spinpolarization is enhanced( Fig. 2(d)) through the regions of different doping levels.

\section{RESULTS AND DISCUSSIONS}

If we consider a certain distribution of charge concentration (all the donors are ionized with $n_{0}^{\uparrow}=n_{d}(x) / 2$ and $n_{0}^{\downarrow}=$ $\left.n_{d}(x) / 2\right)$ and a constant applied electric field as initial conditions, we obtain after solving a system of five differential equations Eqs. (1) with $G^{\sigma}=0,(2)$ and (3) for $t \gg \tau$ that the total density current as well as the electric field profile throughout the semiconductor reach a stationary configuration, i.e. $J(x, t)=J_{0}$ and $E(x, t)=E(x)$. After injecting a Gaussian-like pulse of spin-polarized electrons in the time interval $\delta t$ in a region of space of the sample and letting it to propagate in the bulk we get the transient dynamics of the spin-carrier and current densities by solving the Eqs. (1), (2) and (3) with the previous stationary values as initial conditions. With this ingredients we are able to compute the spinpolarization landscape for carrier and current densities, respectively $\left(n^{\sigma}-n^{\bar{\sigma}}\right) /\left(n^{\sigma}+n^{\bar{\sigma}}\right)$ and $\left(J^{\sigma}-J^{\bar{\sigma}}\right) /\left(J^{\sigma}+J^{\bar{\sigma}}\right)$ in the $n$-GaAs bulk (Figs. 2(a),(b)) and in the $n / n+$ GaAs junction (Fig. 2(c),(d)) cases. From the Figs. 2(a),(d) we observe that current spin-polarization has a slow rise before a subsequent decay that does not appear within the drift-diffusion description. It can be understood because of the temporal derivatives of the current density are included in our approach. By comparing Figs. 2(a) and 2(c) we note a drastic loss of carrier spin-polarization through the interface in the $n / n+\mathrm{GaAs}$ case. In the Fig. 2(c) we see that the polarization of the electronic pulse is not amplified when it crosses the interface. The charge accumulation effect near the interface happens however it take not effect on the spin polarization as it is claimed in the literature [15]. Besides using the standard definition for spin-polarization, i.e. $\left(n^{\sigma}-n^{\bar{\sigma}}\right) /\left(n^{\sigma}+n^{\bar{\sigma}}\right)$ instead of $n^{\sigma}-n^{\bar{\sigma}}$ like appears in the Ref.[15] we account that the charge background doping is physically present all-time which is properly included in the Poisson's equation in order to keep the selfconsistency of the problem. This fall off in the $n+$ region can be explained physically by the presence of more spin- 
carriers from doping than those in the $n$ region. Instead of this we found that the current spin polarization shows an enhancement in the interface due to a constant total background current Fig. 2d.

\section{SUMMARY AND CONCLUSIONS}

We studied the transient dynamics of spin-polarized electron packets STM-tip injected into homogeneous and inhomogeneous $n$-doped GaAs systems. Our approach shows properly in the regime of the ultra-fast phenomena the transient behavior of the spin-polarization for carrier and current densities. In the case of the $n / n+\mathrm{GaAs}$ system it was proved despite the spin-electron polarized packet is amplified at inter- face it has not consequences over the carrier spin- polarization landscape. Instead of this we found that the carrier spin- polarization has a drastic fall off within the $n / n+$ GaAs junction compared with the homogeneous $n$ - GaAs system due to the spin carrier background. Furthermore an enhancement in the current spin-polarization case was observed for both systems following the dynamics of the spin current densities since that the spin current background has not influence in this case.

\section{Acknowledgments}

I gratefully acknowledge helpful discussions with J. C. Egues and F. M. Souza.This work was supported by the CLAF/CNPq.
[1] F. M. Souza and J. C. Egues, Phys. Rev. B 66, 060301(R) (2002).

[2] V. P. Labella et al., Science 292, 1518 (2001).

[3] Note that the relaxation time approximantion here is performed at level of the balance equations. See S. Datta, Quantum Phenomena v. 8, Addison-Wesley 1989.

[4] D. T. Pierce et al., Rev. Sci. Instrum. 51, 478 (1980).

[5] T. Sogawa, et al., Phys. Rev. B 61, 5535 (2000).

[6] I. Žutić et al., Phys. Rev. B 64, 121201 (2001); Phys. Rev. Lett. 88, 066603 (2002).

[7] Z. G. Yu and M. E. Flatté, Phys. Rev. B 66, 235302 (2002).

[8] U. Ravaioli, Semicond. Sci. Technol. 13, 1 (1998).

[9] In the Boltzmann transport equation we have considered the Fermi surface under an electric field in the $x$-direction therefore we integrate the distribution function of particles in the momentum space to obtain for instance the charge density at $(r, t)$ that is uniform in a vicinity $d^{3} r$. The dynamics of the electrical particles described by the physical magnitude $j$ is leaded by the change of the electrical field in the preferential $x$-direction. In case of $n(x, t)$ we are assumed that the charge density is constant in the remaining $y$ and $z$ directions.

[10] This parameter after microscopic treatment appear in the problem of the two current conduction in ferromagnetic metals (A. Fert, J. Phys. C 2(2), 1784 (1969)).

[11] See, W. H. Press, S. A. Teukolsky, W. T. Vetterling and B. P. Flannery, Numerical Recipes in C, the Art of Scientific Computing, (Cambridge University, London, 1996) $2^{\text {nd }}$ edition Ch. 19.

[12] M.T. Portella et al., Appl. Phys. Lett. 60, 2123 (1992).

[13] Spin lifetime at $300 \mathrm{~K}$ for GaAs is up to $100 \mathrm{ps}$. However spin relaxation lifetimes are expected to be shorter for high kinetic energy electrons $(E=-10 \mathrm{KV} / \mathrm{cm})$.

[14] We found that $\tau_{s f}^{*} \gg \tau_{s f}$ can be holded due to extra transport contributions. Here we assume an infinity value for $\tau_{s f}^{*}$ since that it does not modify the results from the qualitative point of view. The influence of this parameter will be matter of discussion in the another full paper.

[15] Y. V. Pershin and V. Privman, Phys. Rev. Lett. 90, 256602 (2003). 